\title{
Rhesus macaques (Macaca mulatta) exhibit the decoy effect in a perceptual discrimination task
}

\author{
Audrey E. Parrish ${ }^{1,2}$ • Theodore A. Evans ${ }^{2}$ - Michael J. Beran ${ }^{1,2}$
}

Published online: 2 April 2015

(C) The Psychonomic Society, Inc. 2015

\begin{abstract}
The asymmetric dominance effect (or decoy effect) is a form of context-dependent choice bias in which the probability of choosing one of two options is impacted by the introduction of a third option, also known as the decoy. Decoy effects are documented widely within the human consumer choice literature, and even extend to preference testing within nonhuman animals. Here, we extended this line of research to a perceptual discrimination task with rhesus monkeys to determine whether decoy stimuli would impact size judgments of rectangular stimuli. In a computerized task, monkeys attempted to choose the larger of two rectangles that varied in size and orientation (horizontally or vertically oriented). In probe trials, a third stimulus (the decoy) was presented that was smaller than the other two rectangles but matched the orientation of one of them. On half of the probe trials, the presented decoy matched the orientation of the larger stimulus, and on the other half, the decoy matched the orientation of the smaller stimulus. Monkeys rarely selected the decoy stimulus. However, their performance (selection of the largest rectangle) increased relative to the baseline trials (with only two choices) when the decoy was congruent in its orientation with the largest rectangle, but decreased relative to baseline when the decoy was incongruent with the largest rectangle. Thus, a decoy stimulus impacted monkeys' perceptual choice behavior even when it was not a viable choice option itself. These results are explained with regard to comparative evaluation mechanisms.
\end{abstract}

Audrey E. Parrish

audrey.parrish1@gmail.com

1 Department of Psychology, Georgia State University, P.O. Box 5010, Atlanta, Georgia 30302-5010

2 Language Research Center, Georgia State University, Atlanta, Georgia
Keywords Asymmetric dominance effect · Decoy effects . Context effects $\cdot$ Choice behavior - Perceptual discrimination . Rhesus macaque $\cdot$ Macaca mulatta

A homebuyer's realtor has helped her narrow down the many real estate options to two choices. The first home is fully renovated and a half-hour drive from work, whereas the second home is unrenovated but a mere 5-min walk from work. The buyer cannot decide between the two options, since she values both home quality and a short commute time. Her realtor introduces a third option, also known as the decoy-a partially renovated home that boasts a lengthy 1 -h commute. Because this option is weaker on both the dimensions of home quality and commute time than the first home, the buyer chooses the first, fully renovated home with the half-hour commute by car. Rational choice theory's condition of regularity states that the relative preferences for the original alternatives within a set should not change with the introduction of a third choice, because each value is made independently of the others (Luce, 1959; Tversky, 1972). However, decisionmaking sometimes is at odds with rational choice theory, and instead, choice behavior may be impacted by context. For the buyer, this so-called decoy option altered her preference for the original two options, such that it increased the preference for the first home that dominated the decoy on both the dimensions of home quality and commute time.

The decoy effect, also known as the asymmetric dominance effect, is a form of context-dependent choice in which the probability of choosing one of two options is impacted by the introduction of a third, weaker option to the choice set (Huber, Payne, \& Puto, 1982). In a choice set, the original two options vary on multiple dimensions, such that the first option dominates the second on one dimension, and the second option dominates the first on a separate dimension. The 
decoy stimulus increases the probability of choosing the original item to which it is most similar. Furthermore, there is a positive correlation between this choice behavior and the similarity of the two items (Huber et al., 1982).

In our example, the first home was stronger in the dimension of home quality, but the second home was stronger in the dimension of commute time. If the homebuyer values both of these dimensions to equal extents, the options are rendered equally attractive, and a decision-maker is indifferent between the two options. The introduction of a decoy stimulus that is similar, but only dominated by one of the original options (and thus is asymmetrically dominated) results in a shift in the preferences for the original options (Huber et al., 1982). In the home example, the buyer's preference for the first home increased with the introduction of the decoy home that was weaker than the first on both the dimensions of quality and commute time. The asymmetric dominance effect is discussed in light of comparative versus absolute evaluations. Within comparative assessments, a decision-maker assigns relative values to an option, which may change as the choice set increases or decreases. This stands in contrast to an absolute mechanism in which an assigned value is made independently of other alternatives, as predicted by the condition of regularity (e.g., Bateson, Healy, \& Hurly, 2002; Luce, 1959).

Although decoy effects have been widely documented in the consumer research literature (e.g., Doyle, O'Connor, Reynolds, \& Bottomley, 1999; Huber et al., 1982; Parducci, 1965; Pettibone \& Wedell, 2000; Wedell, 1991), the phenomenon extends to various forms of human preference testing outside of the consumer domain, including mate-choice behavior (Sedikides, Ariely, \& Olsen, 1999), policy decisions (Herne, 1997), and political races (Pan, O'Curry, \& Pitts, 1995). Moreover, the asymmetric dominance effect has been investigated and demonstrated to varying degrees within several nonhuman animal and insect species in which the addition of a decoy stimulus has resulted in a shift in the preferences for the original options within a choice set. The nonhuman species tested have included ants (Edwards \& Pratt, 2009), honeybees and gray jays (Shafir, Waite, \& Smith, 2002), hummingbirds (Bateson et al., 2002; Hurly \& Oseen, 1999), starlings (Bateson, 2002; Schuck-Paim, Pompilio, \& Kacelnik, 2004), and cats (Scarpi, 2011). For example, Bateson et al. investigated hummingbird decision-making behavior in a foraging paradigm that involved choices between food items (artificial flowers) differing on two dimensions (volume of nectar and sucrose concentration), such that one of the original two options was more valuable in terms of sucrose concentration $(15 \mu \mathrm{l} ; 40 \%$ sucrose $)$ and the second option was more valuable in terms of nectar volume ( $45 \mu \mathrm{l} ; 30 \%$ sucrose). A third stimulus (the decoy) was introduced that was inferior to the first flower in both dimensions ( $10 \mu \mathrm{l} ; 35 \%$ sucrose). The authors documented a shift in the relative preferences for the original items with the introduction of the decoy to the choice set, demonstrating that additional alternatives impacted choice behavior in preference testing with nonhuman animals (Bateson et al., 2002). The authors concluded that, as with humans, animal choice behavior is subject to violations of the regularity condition because evaluations are made comparatively, depending upon context, rather than absolutely. Thus, the impact of a decoy option on decision-making appears to be widespread across multiple human domains, extending to nonhuman species.

Decoy effects are prevalent not only in traditional decisionmaking tasks using preference testing, but also in perceptual discriminations (Choplin \& Hummel, 2005; Trueblood, Brown, Heathcote, \& Busemeyer, 2013; Tversky, 1972). Imagine being offered the choice between a round cake and a square cake that are nearly identical in terms of their total volumes. No clear preference for one over the other would exist. But, then imagine that a third cake, round in shape and smaller than the first round cake, was offered. Now, if a preference emerged for the larger round cake over the square cake, this would be an example of the decoy effect, because the original options within the set were evaluated against a third, weaker option (the decoy) that changed an original indifference to a preference for the member of the original set that dominated the decoy.

Perceptual decoy effects are typically studied using some type of discrimination task in which participants must differentiate stimuli on the basis of physical attributes, such as height and/or width. Performance is then measured after the introduction of an additional alternative, which may enhance the selection of one of the original options. For example, human participants chose between rectangles of variable size, including an asymmetrically dominated decoy that was introduced to enhance preference for one of the two original rectangles (Trueblood et al., 2013). Depending upon the nature of the decoy presented, there was an increase in the probability of selecting the option that the decoy was similar to, the option that it was dissimilar to, and the option that rendered a compromise between the alternatives. The authors discussed their results in light of the preferential-choice literature, in which these three context effects have been documented in higher-level decision-making tasks (Huber et al., 1982; Simonson, 1989; Tversky, 1972).

Although there has been less research with perceptual than with value-driven decoy effects, these studies offer the unique opportunity to explore context effects in basic, perceptualdiscrimination tasks in which decisions regarding stimuli are generated early and quickly. Moreover, studies within the perceptual domain are excellent candidates for use in comparative research with nonhuman animals (especially primates), which have demonstrated psychophysical performance patterns in size-discrimination studies that rival, or even exceed, those in human performance (e.g., Menzel, 1960, 1961; Menzel \& Davenport, 1962; Schmitt, Kröger, Zinner, Call, $\&$ Fischer, 2013). Finally, perceptual tasks of this nature bypass the requirement for language-based instructions or 
extensive training histories or specific forms of knowledge that may impact decision-making strategies or responses.

Although decoy effects have been documented within several nonhuman animal species using preference testing, we wanted to explore whether and how they manifest in a perceptual discrimination task with monkeys. Our objective was to determine whether a decoy stimulus would impact the discrimination performance of rhesus monkeys in a perceptual task to the same extent that has been observed in nonperceptual preference tasks with some animal species (e.g., Bateson, 2002; Bateson et al., 2002; Scarpi, 2011; Schuck-Paim et al., 2004; Shafir et al., 2002). Moreover, we were interested in documenting whether the decoy effect would impact performance in the same direction that has been observed in humans in a size discrimination task in which a similar, but inferior, decoy enhanced the preference for the focal option.

In the present study, we introduced a perceptual sizediscrimination task similar to that used by Trueblood et al. (2013, Exp. 1) with humans. In our study, rhesus monkeys (Macaca mulatta) attempted to choose the larger of two rectangular stimuli that varied in their size and orientation (horizontally or vertically oriented) in each trial. We varied task difficulty to generate a range of difficulties in choosing the larger rectangle, in the hopes that this would also highlight the extent to which a decoy effect could interfere with typical discrimination performance. In baseline trials, only two stimuli were presented - there was no decoy. In probe trials, a third stimulus (the decoy) was presented that was always smaller than the other two rectangles but matched the orientation of one of those rectangles. On half of the probe trials, the presented decoy matched the orientation of the larger stimulus (congruent condition), and on the other half the decoy matched the orientation of the smaller stimulus (incongruent condition). Essentially, the decoy stimulus should have elicited a comparison between itself and the original rectangle in the same orientation. If the decoy effect emerged in the present study, we anticipated that the congruent condition would lead to increased performance (selection of the larger rectangle) relative to the baseline condition, since the same-orientation rectangle was truly the larger of the original set and was comparatively larger than the decoy. Alternatively, the incongruent condition should lead to decreased performance relative to the baseline condition, because the same-orientation rectangle was the smaller of the original set but was still comparatively larger than the decoy.

\section{Experiment 1}

\section{Methods}

Subjects Seven adult male rhesus monkeys between the ages of 11 and 31 years were tested. The monkeys were singly housed for six of the seven days each week, but with continuous visual and auditory access to other monkeys housed in the same colony room. One day per week, each monkey was paired with a socially compatible conspecific in an indooroutdoor enclosure, during which time monkeys did not participate in research projects but instead were free to engage in enrichment activities and interact with each other. When singly housed, monkeys had continuous access to a dedicated computer system for assessing various cognitive capacities (e.g., Agrillo, Parrish, \& Beran, 2014; Beran, Evans, Klein, \& Einstein, 2012; Beran \& Parrish, 2013; Evans \& Beran, 2012; Evans, Perdue, Parrish, \& Beran, 2014; Smith, Coutinho, Church, \& Beran, 2013; Smith, Flemming, Boomer, Beran, \& Church, 2013). These test sessions typically lasted 4-6 h, and monkeys worked or rested at their own choosing throughout these sessions. Monkeys always had access to water and were given a daily meal each afternoon, independent of whether and how much they worked on the computer task.

Apparatus The monkeys were tested using the Language Research Center's Computerized Test System (Fig. 1). This system consisted of a personal computer, digital joystick controller, color monitor, and pellet dispenser (Evans, Beran, Chan, Klein, \& Menzel, 2008; Richardson, Washburn, Hopkins, Savage-Rumbaugh, \& Rumbaugh, 1990). Monkeys manipulated the joystick with their hands to control a small cursor somewhere on the computer screen. Contacting stimuli with the cursor sometimes resulted in the delivery of 94-mg banana-flavored chow pellets (Bio-Serv, Frenchtown, NJ) via a pellet dispenser that was connected to the computer. The task program was written in Visual Basic 6.0.

Design and procedure We presented monkeys with a size discrimination task on a white background. At the outset of each trial, the monkey had to move the cursor upward into

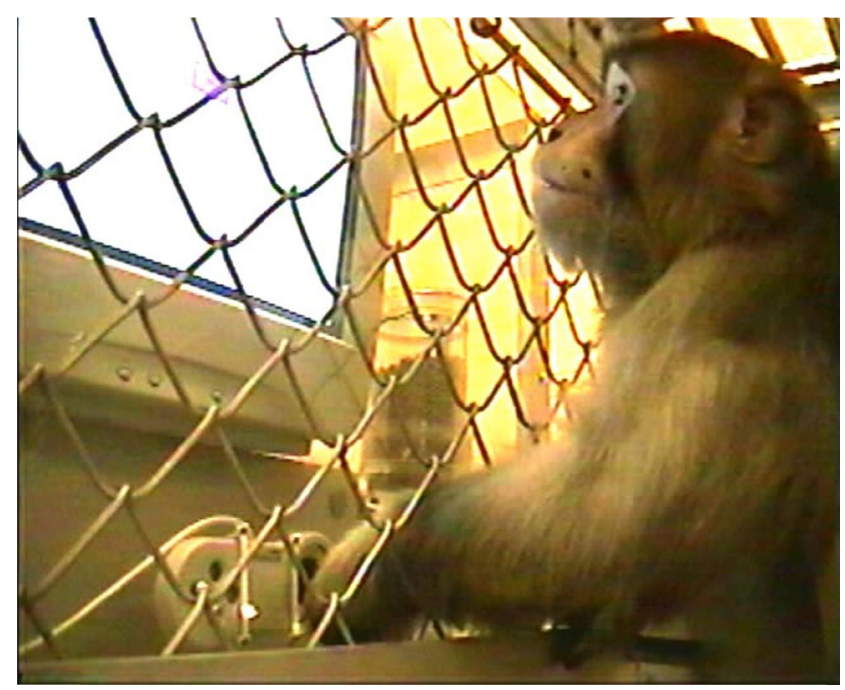

Fig. 1 Experimental setup for the individual test stations at which the monkeys worked on the task. The test stations included a personal computer, monitor, joystick controller, and pellet dispenser 
contact with a gray rectangle at the top center of the computer screen. This was the trial-initiation response. When the rectangle was contacted, it disappeared, and two or three black rectangles appeared at the top of the screen in the left, center, and/or right positions (positions were randomly assigned across trials by the computer program). The monkey then had to move the cursor, which had reappeared in the center of the screen, into contact with one of those rectangles. If the selected rectangle was the largest rectangle on the screen for that trial, the monkey heard a melodic tone and was rewarded with a single food pellet, the screen was cleared, and after a 1-s intertrial interval, the gray rectangle and cursor reappeared at the start of the next trial. Incorrect responses led to a buzz tone, and the screen was cleared for a 20 -s time-out period, during which the screen remained blank before presentation of the gray rectangle at the start of the next trial.

Training sessions During training sessions, monkeys saw only two rectangles on the screen during each trial (Fig. 2). One

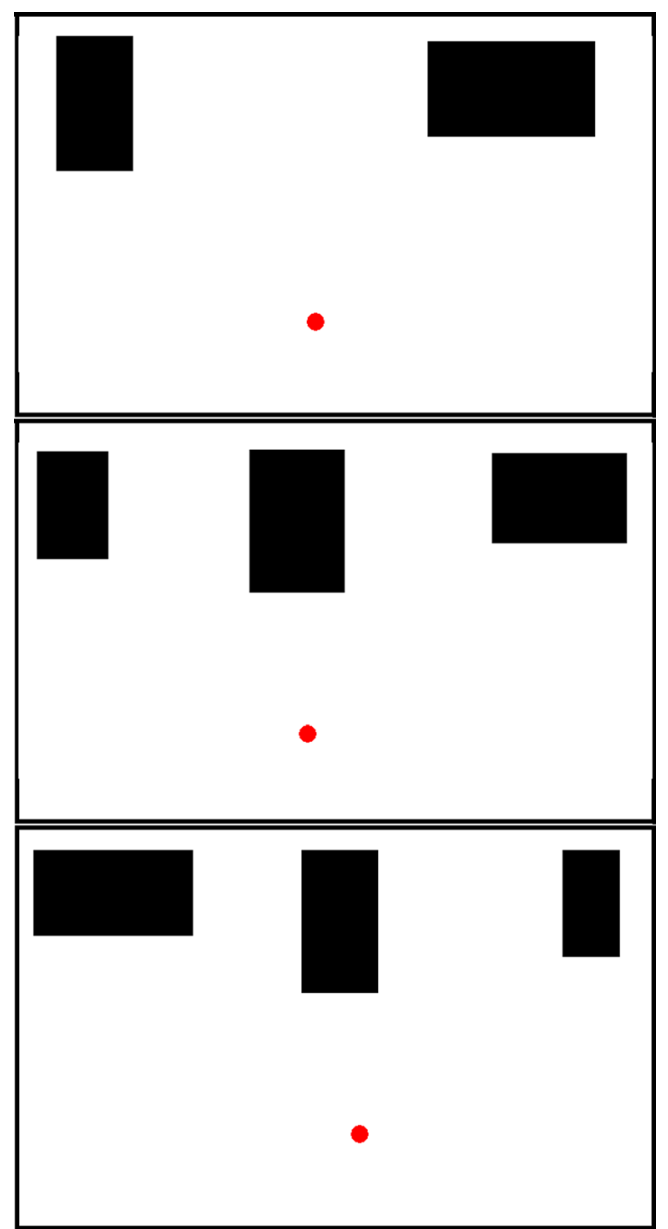

Fig. 2 The testing conditions. The top panel shows a baseline trial (no decoy; correct choice at right), the middle panel shows a congruent trial (decoy in the same orientation as the larger rectangle; correct choice at center), and the bottom panel shows an incongruent trial (decoy in the same orientation as the smaller rectangle; correct choice at left) rectangle was presented such that its height was greater than its width (called the "tall" rectangle), and the other had a width greater than its height (called the "wide" rectangle). On half of these trials, the program generated a relatively small rectangle with a width that was randomly chosen from the range of 120 to 169 pixels and a height randomly chosen from a range of 60 to 109 pixels. On the other half of the trials, the program generated a relatively small rectangle with a height that was randomly chosen from the range of 120 to 169 pixels and a width randomly chosen from a range of 60 to 109 pixels. A second, larger, rectangle was generated with a height that was determined by multiplying the width of the first rectangle by the value $[1+($ Level $* 0.06)]$ and a width that was determined by multiplying the height of the first rectangle by the value [1 $+($ Level * 0.06)]. The level was a randomly chosen value from 1 to 8 on each trial (with Level 1 being the most difficult and Level 8 being the least difficult). Thus, the level acted as an objective value for the degree of difference in size between the two rectangles. These formulas also ensured that on each trial one rectangle was wider than it was tall, and the other was taller than it was wide. The larger rectangle was equally likely to be presented in the tall orientation or the wide orientation, and this was true across all 8 difficulty levels.

Monkeys completed full sessions in which only these control trials with just two rectangle choices were presented until they completed a test session at greater than $70 \%$ accuracy (overall selection of the larger rectangle). Once a monkey had reached criterion on the basic discrimination, the test phase began, in which decoy trials could be presented.

Test sessions In test sessions, three conditions were presented to the monkeys in the same experimental task. In the baseline condition, the trials were identical to those presented in the training phase, and only two choices were presented to the monkey: one rectangle in a tall orientation, and one in a wide orientation. In the other two conditions, however, a third rectangle was presented on the screen in the third available location (Fig. 2). In the congruent condition, this rectangle had the same orientation as the larger of the other two rectangles on the screen. In the incongruent condition, this rectangle had the same orientation as the smaller of the other two rectangles on the screen. In both cases, this third rectangle (the decoy) was smaller than its same-orientation counterpart, with a width and a height that were only $75 \%$ that of the focal option (i.e., its counterpart of the same orientation). Thus, if the decoy effect occurred, and this item led monkeys to see the counterpart as larger than it actually was, then performance would be enhanced in the congruent condition because the alreadylargest rectangle on the screen would benefit from being made to look larger by its decoy. However, in the incongruent condition, the decoy would make the smaller of the other two rectangles look larger than it really was, and perhaps dampen performance relative to the baseline condition. 
During test sessions, baseline trials were presented with a probability of .70, and congruent and incongruent trials each were presented with a probability of .15. Trial level was randomly selected from the range of 1 to 8 for all three conditions. As in the training phase, selection of the largest rectangle onscreen led to a food reward and presentation of the next trial, whereas selection of a rectangle that was not the largest onscreen led to a 20 -s time out before presentation of the next trial. All monkeys were scheduled to complete 3,000 trials in the test phase. However, because of a program error, three monkeys, Murph, Hank, and Gale, only completed 2,000 trials.

\section{Results}

During the test phase, selection of the decoy stimulus among the three choices was a very rare outcome: for Chewie, $0.5 \%$ of trials; for Gale, $0.2 \%$ of trials; for Hank, $1.0 \%$ of trials; for Lou, $0.4 \%$ of trials; for Luke, $0.4 \%$ of trials; for Murph, $0.3 \%$ of trials; for Obi, $0.07 \%$ of trials. Thus, the decoy rectangle was not a viable choice option to the monkeys, since they rarely $(\leq 1.0 \%)$ selected this option.

Prior to the analyses, the data from the eight difficulty levels were binned into four levels by combining pairs of levels. Thus, Levels 1-2 became the new Level 1, Levels 34 became the new Level 2, Levels 5-6 became the new Level 3 , and Levels 7-8 became the new Level 4 .

Figure 3 shows the mean percentages of trials correct at each binned stimulus level for each condition and each orientation. A within-subjects repeated measures analysis of variance (ANOVA) was conducted to examine the effects of binned level (1-4), condition (baseline, congruent, incongruent), and correct stimulus orientation (tall, wide) on the selection of the larger rectangle. We found significant main effects of level, $F(3,18)=48.23, p<.001, \eta_{\mathrm{p}}{ }^{2}=.89$, condition, $F(2$, $12)=7.77, p=.007, \eta_{\mathrm{p}}{ }^{2}=.56$, and orientation, $F(1,6)=$ $10.99, p=.016, \eta_{\mathrm{p}}{ }^{2}=.65$. There was also a significant interaction of level and condition, $F(6,36)=4.11, p=.003, \eta_{\mathrm{p}}{ }^{2}=$ .41 , indicating that performance diverged more between conditions at the more difficult levels than at the easier levels. A significant interaction of orientation and condition emerged, $F(2,12)=5.29, p=.023, \eta_{\mathrm{p}}{ }^{2}=.47$, but no interaction of orientation and level, $F(3,18)=2.10, p=.14, \eta_{\mathrm{p}}{ }^{2}=.26$, and no three-way interaction, $F(6,36)=0.59, p=.74, \eta_{\mathrm{p}}{ }^{2}=.09$.

To examine the interaction between level and condition, we compared each condition to each of the others (baseline vs. congruent, baseline vs. incongruent, and congruent vs. incongruent) at each level $(1,2,3,4)$ using a paired-samples $t$ test. A Bonferroni-adjusted alpha level of .017 was used per test (.05/ $3)$. Performance did not differ significantly for any conditions at Level 1 [baseline vs. congruent, $t(6)=-1.55, p=.17$; baseline vs. incongruent, $t(6)=2.52, p=.04$; congruent vs. incongruent, $t(6)=2.42, p=.05]$. For Level 2, performance was
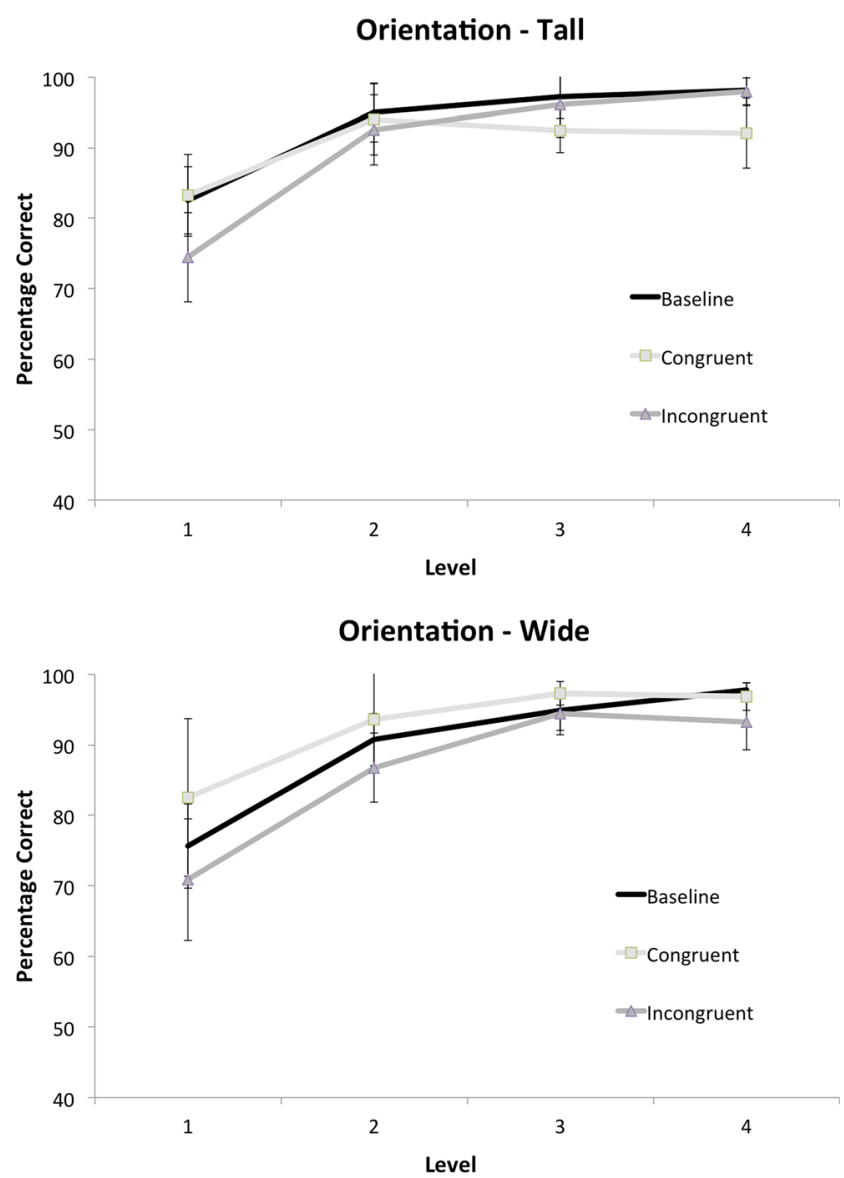

Fig. 3 Mean percentages of trials correct at each binned stimulus level (1-4, with Level 1 being the objectively most difficult and Level 4 being the objectively least difficult discrimination) for each condition (baseline, congruent, and incongruent) and each orientation (tall and wide) in Experiment 1. Errors bars represent $95 \%$ confidence intervals

significantly higher in the congruent condition than in the incongruent condition $[t(6)=3.74, p=.01]$, but did not differ between baseline and incongruent $[t(6)=3.22, p=.018]$, nor between baseline and congruent $[t(6)=-0.76, p=.48]$. Performance did not differ significantly for any conditions at Level 3 [baseline vs. congruent, $t(6)=1.02, p=.35$; baseline vs. incongruent, $t(6)=$ $0.99, p=.36$; congruent vs. incongruent, $t(6)=-0.25, p=.81]$. For Level 4, performance was significantly higher in the congruent condition than in the baseline condition $[t(6)=3.33$, $p=.016]$, but did not differ between the baseline and incongruent conditions $[t(6)=2.59, p=.04]$, nor between the congruent and incongruent conditions $[t(6)=-1.13, p=.30]$.

To explore the interaction of orientation and condition, we compared the effects of orientation in each condition, collapsing across levels using a paired-samples $t$ test. A Bonferroni-adjusted alpha level of .017 was used per test $(.05 / 3)$. Performance was significantly higher in the tallbaseline condition than in the wide-baseline condition $[t(6)=4.05, p=.007]$, indicating that when the correct stimulus was in the tall orientation, monkeys performed better. Performance was significantly higher in the tall- 
incongruent condition than in the wide-incongruent condition $[t(6)=3.45, p=.014]$, also confirming that performance was better when the correct stimulus was in a tall orientation. Performance did not differ in the tall-congruent versus widecongruent conditions $[t(6)=-1.21, p=.274]$.

We also explored whether response times to make a choice within a trial varied between the conditions, and we examined this for correct and incorrect trials (Fig. 4), under the assumption that such an effect of condition was more likely to emerge for correctly completed trials, in which animals ultimately made the correct response. Thus, we conducted a two-way repeated measures ANOVA with outcome and condition as variables. First, we removed any response times that exceeded $10 \mathrm{~s}$, which we classified as extreme outliers (they comprised less than $1.3 \%$ of the trials for all of the monkeys), and then calculated the mean response times for each monkey in each condition for correct and incorrect trials. There was not a significant effect of condition on response times: $F(2,12)=$ $0.52, p=.61, \eta_{\mathrm{p}}{ }^{2}=.08$. There also was not a significant effect of outcome on response times: $F(1,6)=5.6, p=.06, \eta_{\mathrm{p}}{ }^{2}=.48$. However, there was a significant interaction between condition and outcome, $F(2,12)=6.33, p=.013, \eta_{\mathrm{p}}{ }^{2}=.51$.

To explore this interaction, we conducted separate repeated measures ANOVAs for correct and incorrect trials, comparing the response times in each condition. We found a significant effect of condition for correct trials, $F(2,12)=$ $60.3, p<.001, \eta_{\mathrm{p}}{ }^{2}=.91$, but not for incorrect trials, $F(2,12)$ $=2.2, p=.15, \eta_{\mathrm{p}}{ }^{2}=.27$. We then compared each condition to the others for correct trials, using paired-samples $t$ tests and a Bonferroni-corrected alpha level of .017, given the three comparisons. Response times were significantly faster for congruent correct trials than for baseline correct trials $[t(6)=$ $3.58, p=.01]$, and significantly faster for congruent correct trials than for incongruent correct trials $[t(6)=-8.26, p<$ $.001]$. Response times were also significantly faster for baseline correct trials than for incongruent correct trials $[t(6)=-11.64, p<.001]$. These results indicate that the

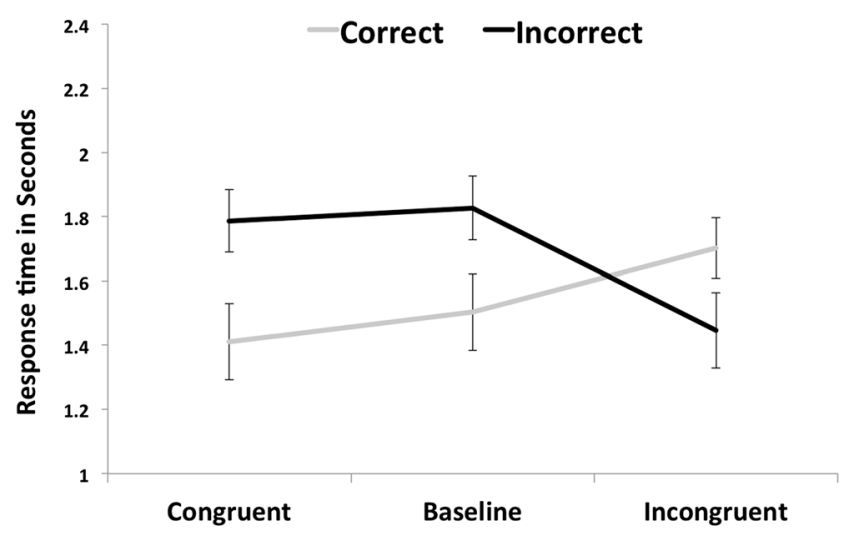

Fig. 4 Mean response times for each condition for correctly completed and incorrectly completed trials in Experiment 1. Error bars show standard errors of the means monkeys responded fastest in congruent trials and slowest in incongruent trials when they made a correct response, a pattern consistent with the idea that congruent decoy stimuli aided and speeded choice behavior, whereas incongruent decoy stimuli hurt and slowed choice behavior.

In considering individual differences among the monkeys, we collapsed across orientations and analyzed the performance (percentages correct) of each monkey in each condition (baseline, congruent, and incongruent) across all levels. Furthermore, we analyzed whether each condition differed from the others for each monkey at each level by using a chi square or Fischer's exact test on the frequency data for correct/incorrect choices. These results are presented in Table 1. Although not every condition varied from every other condition at each level, when a significant difference did occur, it supported our hypotheses that congruent performance would be higher than baseline performance, incongruent performance would be lower than baseline performance, and congruent performance would be higher than incongruent performance.

Table 1 Individual results from Experiment 1, including the percentages correct for each animal in each condition (baseline, congruent, and incongruent) and for each level

\begin{tabular}{|c|c|c|c|c|c|}
\hline & & Level 1 & Level 2 & Level 3 & Level 4 \\
\hline \multirow[t]{3}{*}{ Chewie } & Baseline & 83.17 & 95.97 & 96.60 & 97.76 \\
\hline & Congruent & 89.22 & 96.54 & 93.94 & 94.76 \\
\hline & Incongruent & 85.96 & $90.46^{*}$ & 96.07 & 93.87 \\
\hline \multirow[t]{3}{*}{ Gale } & Baseline & 77.08 & 95.77 & 98.85 & 98.17 \\
\hline & Congruent & $92.05^{*}$ & 96.30 & 97.06 & $90.63 *$ \\
\hline & Incongruent & $65.34^{\wedge}$ & $89.74 *$ & $94.00 *$ & $91.46^{*}$ \\
\hline \multirow[t]{3}{*}{ Hank } & Baseline & 68.22 & 82.07 & 87.79 & 94.61 \\
\hline & Congruent & 61.54 & 76.52 & 92.03 & 88.47 \\
\hline & Incongruent & 69.27 & 76.97 & 85.82 & 94.82 \\
\hline \multirow[t]{3}{*}{ Lou } & Baseline & 80.92 & 93.36 & 96.97 & 99.40 \\
\hline & Congruent & 86.46 & 94.00 & $90.89 *$ & $95.21^{*}$ \\
\hline & Incongruent & $74.39^{\wedge}$ & 91.66 & $97.56^{\wedge}$ & $96.23^{*}$ \\
\hline \multirow[t]{3}{*}{ Luke } & Baseline & 81.03 & 94.56 & 98.15 & 98.13 \\
\hline & Congruent & 82.02 & 97.88 & 96.67 & 99.14 \\
\hline & Incongruent & 77.25 & $90.29^{\wedge}$ & 100.00 & 96.69 \\
\hline \multirow[t]{3}{*}{ Murph } & Baseline & 74.57 & 90.75 & 95.46 & 98.22 \\
\hline & Congruent & 76.79 & 95.45 & 94.60 & 95.54 \\
\hline & Incongruent & 61.21 & 90.28 & 94.54 & 97.14 \\
\hline \multirow[t]{3}{*}{ Obi } & Baseline & 88.43 & 97.58 & 98.84 & 99.60 \\
\hline & Congruent & 92.01 & 100.00 & 99.09 & 97.50 \\
\hline & Incongruent & $75.17^{* \wedge}$ & 98.15 & 99.06 & 99.19 \\
\hline
\end{tabular}

Asterisks indicate $p<.05$ for comparisons of the congruent or incongruent condition to the baseline condition. Carets indicate $p<.05$ for the comparison of the congruent to the incongruent condition. 


\section{Discussion}

The decoy stimulus moderately impacted perceptual choice behavior among rhesus monkeys in the present experiment, even when the decoy stimulus itself did not represent a viable choice option. As expected, the monkeys were highly successful in choosing the larger of two rectangular stimuli within the present experiment, with performance increasing as the true difference between the two stimuli increased. Performance was impacted by the presence of a decoy stimulus, serving either to increase (congruent condition) or decrease (incongruent condition) the selection of the larger rectangle, relative to baseline levels. This pattern was more pronounced for the most difficult levels and for the wide orientation. The individual results also indicated that all cases of a significant difference between any two conditions occurred in the direction indicative of a decoy effect.

The decoy stimuli also affected response times. When the decoy was congruent, monkeys made correct choices even faster than when no decoy stimulus was present. However, the reverse was true for incongruent decoys. When those decoys were present, even when monkeys made the correct choice, they took longer to do so. These results also support the idea that decoys affect how monkeys view and respond to choices that include a nonviable decoy option.

In exploring the interaction between condition and orientation, we confirmed that performance levels were higher when the correct choice was in the tall orientation rather than the wide orientation in the baseline and incongruent conditions. The rhesus monkeys chose more accurately when they were supposed to choose the vertical rather than the horizontal stimulus. In the incongruent condition, the decoy effect was less pronounced for vertical stimuli than for horizontal stimuli. Thus, the monkeys were less likely to erroneously choose the horizontal foil stimulus even when a horizontal decoy was present. These results support the horizontal-vertical illusion, in which a vertically oriented line appears longer than an identical but horizontally oriented line, which has been documented in several nonhuman primate species (Cercopithecinae and Cebus: Dominguez, 1954; Harris, 1968). The present results provide support that rhesus monkeys may also overestimate vertical dimensions relative to horizontal ones, serving to insulate the impact of horizontally oriented decoy stimuli.

In general, the monkeys performed at very high levels, and this created a potential ceiling effect that might have masked a stronger decoy effect. This was confirmed by the level $\times$ condition interaction. Thus, we conducted a second experiment in which we restricted the trial levels to the more difficult half of the continuum used in Experiment 1, to better assess the prevalence of perceptual decoy effects in these monkeys' discrimination performances.

\section{Experiment 2}

\section{Methods}

Subjects and apparatus These were the same as in Experiment 1 .

Design and procedure There was no training phase in this experiment; all monkeys began in the test phase, which was functionally identical to that in Experiment 1. The only difference pertained to the difficulty levels of trials, which were increased across the full range of eight levels. Now the second, larger rectangle was generated with a height that was determined by multiplying the width of the first rectangle by the value $[1+($ Level $* 0.015)]$ and a width that was determined by multiplying the height of the first rectangle by the value [ 1 $+($ Level $* 0.015)]$. Note that the multiplier of 0.015 was $1 / 4$ the size of the multiplier from Experiment 1 (0.06). The same three conditions were presented in the same proportions as in Experiment 1, and each monkey completed 3,000 trials.

\section{Results}

Again, selection of the decoy stimulus among the three choices was a very rare outcome ( $\leq 1.0 \%)$ : Chewie, $0.4 \%$ of trials; Gale, $0.2 \%$ of trials; Hank, $1.0 \%$ of trials; Lou, $0.07 \%$ of trials; Luke, $0.03 \%$ of trials; Murph, $0.9 \%$ of trials; Obi, $0.2 \%$ of trials.

The data again were binned from eight difficulty levels into four levels prior to analysis. Figure 5 shows the mean percentages of trials correct at each binned stimulus level for each condition and each orientation. We conducted a withinsubjects ANOVA to examine the effects of level (1-4), condition (baseline, congruent, incongruent), and orientation (tall, wide) on the selection of the larger rectangle in Experiment 2. Significant main effects emerged of level, $F(3,18)=108.93, p$ $<.001, \eta_{\mathrm{p}}{ }^{2}=.95$, and condition, $F(2,12)=14.58, p=.001, \eta_{\mathrm{p}}{ }^{2}$ $=.71$. However, there was no longer an effect of orientation, $F(1,6)=.001, p=.973, \eta_{\mathrm{p}}{ }^{2}<.01$, nor any significant interactions.

As in Experiment 1, we analyzed the response time data to determine whether the latency to make a choice varied as a function of condition, with a two-way repeated measures ANOVA comparing the average response times in the baseline, congruent, and incongruent conditions for correct and incorrect trials. Again, we removed any response times that exceeded $10 \mathrm{~s}$, which we classified as extreme outliers. Such outliers were very rare, comprising less than $1.2 \%$ of trials for all of the monkeys. We found significant effects of condition, $F(2,12)=5.72, p=.02, \eta_{\mathrm{p}}{ }^{2}=.49$, and outcome, $F(1,6)=$ $57.72, p<.001 \eta_{\mathrm{p}}{ }^{2}=.91$, on response times, and again there was a significant interaction between condition and outcome, $F(2,12)=62.07, p<.001, \eta_{\mathrm{p}}{ }^{2}=.91$. 


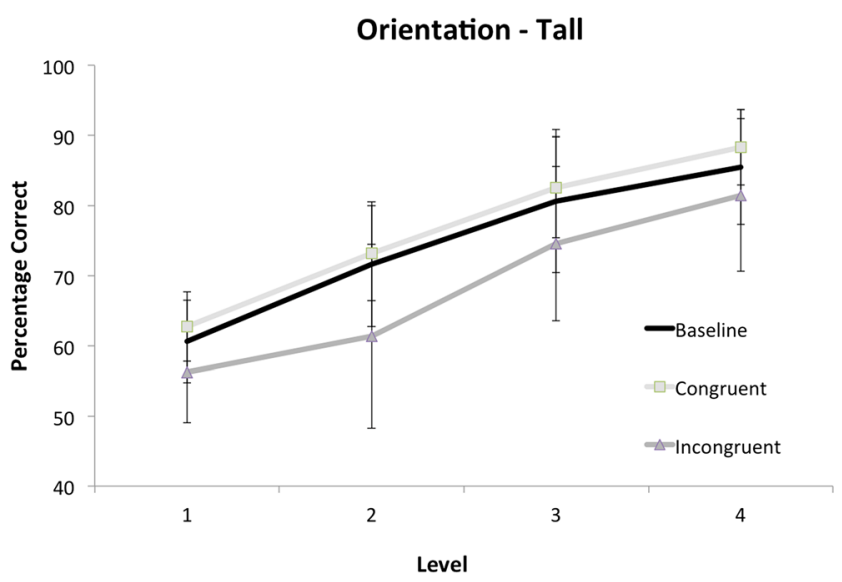

Orientation - Wide

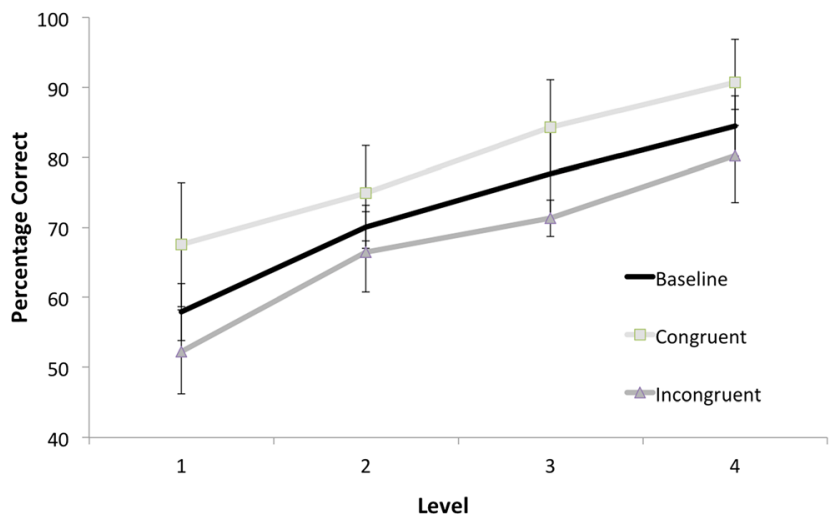

Fig. 5 Mean percentages of trials correct at each binned stimulus level for each condition (baseline, congruent, and incongruent) and each orientation (tall and wide) in Experiment 2. Errors bars represent $95 \%$ confidence intervals

As in Experiment 1, we next conducted separate repeated measures ANOVAs for correct and incorrect trials, comparing the response times in each condition (Fig. 6). We found significant effects of condition for correct trials, $F(2,12)=$ $76.09, p<.001, \eta_{\mathrm{p}}^{2}=.93$, and for incorrect trials, $F(2,12)=$ $27.99, p<.001, \eta_{\mathrm{p}}{ }^{2}=.82$. We then compared each condition to the others using paired-samples $t$ tests for correct and incorrect trials (using a Bonferroni-corrected $p$ value of .017). For correct trials, response times were significantly faster for congruent than for baseline correct trials $[t(6)=5.71, p=.001]$, and significantly faster for congruent than for incongruent correct trials $[t(6)=-10.42, p<.001]$. Response times also were significantly faster for baseline than for incongruent correct trials $[t(6)=$ $-7.52, p<.001]$. For incorrect trials, response times were significantly slower for congruent than for incongruent trials $[t(6)=5.61, p=.001]$, and significantly slower for congruent than for baseline trials $[t(6)=-9.0, p<.001]$. As in Experiment 1 , these results indicate that the monkeys responded fastest in the congruent trials and slowest in incongruent trials when they made a correct response. However, monkeys responded slowest in congruent trials when they made an incorrect response.

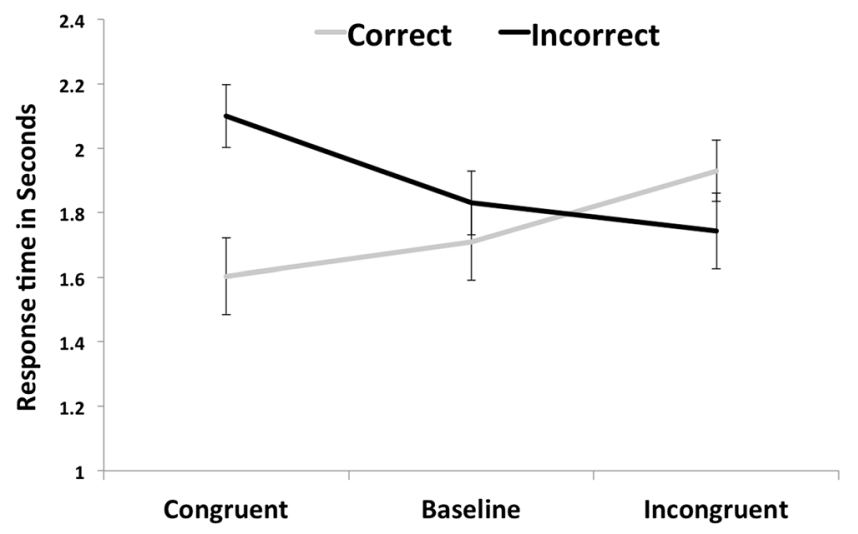

Fig. 6 Mean response times for each condition for correctly completed and incorrectly completed trials in Experiment 2. Error bars show standard errors of the means

Additionally, we analyzed the individual data for all monkeys in Experiment 2 in the same manner as in Experiment 1, and these results are presented in Table 2. As in Experiment 1, although not every condition varied from every other condition at each level for each monkey, whenever a significant difference occurred, it supported our hypotheses that congruent performance would be higher than baseline performance, incongruent performance would be lower than baseline performance, and congruent performance would be higher than incongruent performance.

\section{Discussion}

As in Experiment 1, rhesus monkeys' perceptual judgments about rectangle size were impacted by the presence of a decoy stimulus. However, the introduction of more difficult discriminations (via levels that spanned only half of the original continuum) eliminated the interaction between condition and level. This interaction in Experiment 1 was likely due to high levels of performance in the easier levels within Experiment 1, resulting in a ceiling effect that masked a more pronounced decoy effect. In Experiment 2, the decoy stimulus impacted performance more consistently across levels, either serving to increase (congruent condition) or decrease (incongruent condition) performance relative to the baseline condition (see Fig. 5). Additionally, there was no longer an effect of orientation, indicating that the monkeys were equally proficient across tall and wide trials. This finding was likely due to experience in performing the task and receiving feedback about their responses. Thus, experience diminished the generally higher performance when tall stimuli were the correct choice versus wide stimuli, but the effect of level remained (as was expected, given that this was the objective factor for trial difficulty), and the decoy effect remained, as evidenced by the continued main effect of condition. Thus, across the two experiments, the decoy effect was present, although there were individual differences in the degrees to which this effect was evident. 
Table 2 Individual results from Experiment 2, including the percentages correct for each animal in each condition (baseline, congruent, and incongruent) and for each level

\begin{tabular}{|c|c|c|c|c|c|}
\hline & & Level 1 & Level 2 & Level 3 & Level 4 \\
\hline \multirow[t]{3}{*}{ Chewie } & Baseline & 61.63 & 74.37 & 85.70 & 90.53 \\
\hline & Congruent & 64.00 & 78.80 & 85.10 & 95.99 \\
\hline & Incongruent & 63.11 & 68.16 & $76.99 *$ & $88.11^{\wedge}$ \\
\hline \multirow[t]{3}{*}{ Gale } & Baseline & 58.11 & 70.39 & 77.92 & 85.27 \\
\hline & Congruent & $75.04 *$ & 75.91 & $89.25^{*}$ & 90.72 \\
\hline & Incongruent & $59.16^{\wedge}$ & $57.84^{* \wedge}$ & $69.75^{\wedge}$ & $80.70^{\wedge}$ \\
\hline \multirow[t]{3}{*}{ Hank } & Baseline & 55.71 & 59.89 & 60.55 & 69.56 \\
\hline & Congruent & 53.70 & 63.35 & 66.17 & 78.16 \\
\hline & Incongruent & 50.95 & 50.81 & 59.85 & $64.73^{\wedge}$ \\
\hline \multirow[t]{3}{*}{ Lou } & Baseline & 60.44 & 73.92 & 81.64 & 88.52 \\
\hline & Congruent & 72.01 & 80.00 & 89.09 & 94.54 \\
\hline & Incongruent & $49.59^{* \wedge}$ & $56.30^{* \wedge}$ & $70.21^{* \wedge}$ & $82.45^{\wedge *}$ \\
\hline \multirow[t]{3}{*}{ Luke } & Baseline & 59.05 & 73.04 & 80.86 & 87.43 \\
\hline & Congruent & 63.47 & 69.46 & 83.18 & 91.13 \\
\hline & Incongruent & 58.57 & 80.77 & 81.66 & 90.43 \\
\hline \multirow[t]{3}{*}{ Murph } & Baseline & 55.62 & 64.54 & 74.42 & 80.12 \\
\hline & Congruent & 56.76 & 66.32 & 81.48 & 79.86 \\
\hline & Incongruent & $42.42 * \wedge$ & 55.62 & 68.34 & $70.75^{*}$ \\
\hline \multirow[t]{3}{*}{ Obi } & Baseline & 64.23 & 80.00 & 93.13 & 93.67 \\
\hline & Congruent & 70.99 & 84.61 & 89.92 & 96.32 \\
\hline & Incongruent & $55.82^{\wedge}$ & 78.02 & $83.92 *$ & 88.91 \\
\hline
\end{tabular}

Asterisks indicate $p<.05$ for comparisons of the congruent or incongruent condition to the baseline condition. Carets indicate $p<.05$ for the comparison of the congruent to the incongruent condition.

For correctly completed trials, we replicated the response time effect of Experiment 1. However, we also found a different response time pattern for incorrectly completed trials, suggesting that the response times when monkeys were incorrect might have reflected some of the response competition they felt when faced with a decoy stimulus. We discuss this in more detail in the next section.

\section{General discussion}

The present work provides evidence for the asymmetric dominance effect (also known as the decoy effect) within the perceptual domain among rhesus monkeys. Monkeys' perceptual discrimination in the present size judgment task was impacted by the presence of a third, smaller decoy stimulus. A smaller, decoy rectangle enhanced selection of the original rectangle that it was most similar to in terms of its orientation, serving to increase performance relative to baseline if the decoy was in the same orientation as the largest rectangle. Alternatively, this effect disrupted performance relative to baseline if the sameorientation rectangle was the smaller of the two original options. These findings complement similar work documenting the asymmetric dominance effect using traditional preference testing in nonhuman species (Bateson, 2002; Bateson et al., 2002; Hurly \& Oseen, 1999; Scarpi, 2011; Schuck-Paim et al., 2004; Shafir et al., 2002). Furthermore, these results are consistent with human evidence of the decoy effect within the perceptual domain (Choplin \& Hummel, 2005; Trueblood et al., 2013; Tversky, 1972).

Evidence for a perceptual decoy effect in a monkey species supports the notion that context-dependent choice behavior extends beyond higher-order decision-making tasks and emerges during more basic perceptual processing (Trueblood et al., 2013). The decoy stimulus itself was not a viable choice option, since it always was the smallest rectangle within the set, and it was rarely selected in any experiment. Thus, the decoy impacted choice behavior by creating a contrasting option to which the original options were compared. These comparative evaluations are dependent upon the availability and properties of alternatives within a choice set (Bateson et al., 2002). Comparative evaluations may serve to decrease the complexity of decisions by allowing the decision-maker to assign a relative value based on one dimension, rather than constructing an absolute value for each option within a set (Ariely \& Wallsten, 1995). Bateson and colleagues discussed the adaptive value of comparative mechanisms in terms of their potentially lower computational requirements, in relation to more costly absolute evaluations (see Tversky, 1969).

This notion is supported by the difference in the response time data in the present study. On correctly completed trials, the monkeys responded fastest when a congruent (or helpful) decoy was present, but incurred a time cost when an incongruent (or hurtful) decoy was present, even though they still made the correct choice. The decoy stimulus created a contrasting option to the stimulus that it was most similar to; this either quickly led to the correct answer (congruent trials) or instead slowed response time in making the right choice, if the decoy matched the incorrect answer (incongruent trials). In Experiment 2, an additional feature of the response times emerged: when monkeys ultimately made a mistake (and chose the smaller item of the nondecoy pair), they took longest to make this error when the decoy was congruent with the correct choice. In other words, the monkeys likely noticed the decoy that would have helped performance, because it slowed their eventual choice of the wrong answer, the one that was dissimilar to the decoy. Future research that varies the physical proximity or degree of similarity between the decoy and the focal option would be of interest to understanding the limits of this type of contrast effect in monkeys and the resulting impact on response times.

These findings open future avenues for testing the impacts of decoy stimuli on choice behavior in nonverbal species and among preverbal children. Perceptual tasks of this nature require little training or verbal instruction that might impact 
decision-making strategies, making this paradigm accessible to many species and populations. Such tasks easily contrast performance against well-established psychophysical discriminations from baseline conditions and allow for parametric variations of the stimuli, including the decoys. Parametric variations would be useful if one were interested in assessing the ideal decoy characteristics for enhancing or disrupting discrimination performance. The use of basic perceptual discrimination tasks allows for spontaneous and early-emerging effects, such as the decoy effect, to highlight the mechanisms that underlie perceptual responding and context-dependent choice behavior. That monkeys showed these effects indicates that they are evolutionarily shared and not language-dependent, and perhaps may be quite widespread among animals. If this is true, it would suggest that some decisional biases likely have roots in foundational perceptual processes with long evolutionary histories.

Author note Support for this research was provided by NICHD (HD060563), a 2CI Primate Social Cognition, Evolution \& Behavior Fellowship, and the Duane M. Rumbaugh Fellowship from Georgia State University. We thank the animal care and enrichment staff for maintaining the health and wellbeing of the primates and making this research possible, and David A. Washburn for the photograph of the monkey working on the computer.

\section{References}

Agrillo, C., Parrish, A. E., \& Beran, M. J. (2014). Do rhesus monkeys (Macaca mulatta) perceive the Zöllner illusion? Psychonomic Bulletin \& Review, 21, 986-994. doi:10.3758/s13423-013-0573-2

Ariely, D., \& Wallsten, T. S. (1995). Seeking subjective dominance in multidimensional space: An explanation of the asymmetric dominance effect. Organizational Behavior and Human Decision Processes, 63, 223-232.

Bateson, M. (2002). Context-dependent foraging choices in risk-sensitive starlings. Animal Behaviour, 64, 251-260.

Bateson, M., Healy, S. D., \& Hurly, T. A. (2002). Irrational choices in hummingbird foraging behaviour. Animal Behaviour, 63, 587-596.

Beran, M. J., Evans, T. A., Klein, E. D., \& Einstein, G. O. (2012). Rhesus monkeys (Macaca mulatta) and capuchin monkeys (Cebus apella) remember future responses in a computerized task. Journal of Experimental Psychology: Animal Behavior Processes, 38, 233-243.

Beran, M. J., \& Parrish, A. E. (2013). Visual nesting of stimuli affects rhesus monkeys' (Macaca mulatta) quantity judgments in a bisection task. Attention, Perception, \& Psychophysics, 75, 1243-1251.

Choplin, J. M., \& Hummel, J. E. (2005). Comparison-induced decoy effects. Memory \& Cognition, 33, 332-343. doi:10.3758/ BF03195321

Dominguez, K. E. (1954). A study of visual illusions in the monkey. Journal of Genetic Psychology, 85, 105-127.

Doyle, J. R., O’Connor, D. J., Reynolds, G. M., \& Bottomley, P. A. (1999). The robustness of the asymmetrically dominated effect: Buying frames, phantom alternatives, and in-store purchases. Psychology and Marketing, 16, 225-243.

Edwards, S. C., \& Pratt, S. C. (2009). Rationality in collective decisionmaking by ant colonies. Proceedings of the Royal Society B, 276, 3655-3661.
Evans, T. A., \& Beran, M. J. (2012). Monkeys exhibit prospective memory in a computerized task. Cognition, 125, 131-140.

Evans, T. A., Beran, M. J., Chan, B., Klein, E. D., \& Menzel, C. R. (2008). An efficient computerized testing method for the capuchin monkey (Cebus apella): Adaptation of the LRC-CTS to a socially housed nonhuman primate species. Behavior Research Methods, 40, 590-596. doi:10.3758/BRM.40.2.590

Evans, T. A., Perdue, B. M., Parrish, A. E., \& Beran, M. J. (2014). Working and waiting for better rewards: Self-control in two monkey species (Cebus apella and Macaca mulatta). Behavioural Processes, 103, 236-242. doi:10.1016/j.beproc.2014.01.001

Harris, A. V. (1968). Perception of the horizontal-vertical illusion in stumptail monkeys. Radford Review, 22, 61-72.

Herne, K. (1997). Decoy alternatives in policy choices: Asymmetric domination and compromise effects. European Journal of Political Economy, 13, 575-589.

Huber, J., Payne, J. W., \& Puto, C. (1982). Adding asymmetrically dominated alternatives: Violations of regularity and the similarity hypothesis. Journal of Consumer Research, 9, 90-98.

Hurly, T. A., \& Oseen, M. D. (1999). Context-dependent, risk-sensitive foraging preferences in wild rufous hummingbirds. Animal Behaviour, 58, 59-66.

Luce, R. D. (1959). Individual choice behavior: A theoretical analysis. New York, NY: Wiley.

Menzel, E. W., Jr. (1960). Selection of food by size in the chimpanzee and comparison with human judgments. Science, 131, 1527-1528.

Menzel, E. W., Jr. (1961). Perception of food size in the chimpanzee. Journal of Comparative and Physiological Psychology, 54, 588591.

Menzel, E. W., Jr., \& Davenport, R. K., Jr. (1962). The effects of stimulus presentation variable upon chimpanzee's selection of food by size. Journal of Comparative and Physiological Psychology, 55, 235239.

Pan, Y., O'Curry, S., \& Pitts, R. (1995). The attraction effect and political choice in two elections. Journal of Consumer Psychology, 4, 85101.

Parducci, A. (1965). Category judgment: A range-frequency model. Psychological Review, 72, 407-418. doi:10.1037/h0022602

Pettibone, J. C., \& Wedell, D. H. (2000). Examining models of nondominated decoy effects across judgment and choice. Organizational Behavior and Human Decision Processes, 81, 300-328.

Richardson, W. K., Washburn, D. A., Hopkins, W. D., SavageRumbaugh, E. S., \& Rumbaugh, D. M. (1990). The NASA/LRC Computerized Test System. Behavior Research Methods, Instruments, \& Computers, 22, 127-131.

Scarpi, D. (2011). The impact of phantom decoys on choices in cats. Animal Cognition, 14, 127-136.

Schmitt, V., Kröger, I., Zinner, D., Call, J., \& Fischer, J. (2013). Monkeys perform as well as apes and humans in a size discrimination task. Animal Cognition, 16, 829-838.

Schuck-Paim, C., Pompilio, L., \& Kacelnik, A. (2004). State-dependent decisions cause apparent violations of rationality in animal choice. PLoS Biology, 2, e402. doi:10.1371/journal.pbio.0020402

Sedikides, C., Ariely, D., \& Olsen, N. (1999). Contextual and procedural determinants of partner selection: Of asymmetric dominance and prominence. Social Cognition, 17, 118-139.

Shafir, S., Waite, T. A., \& Smith, B. H. (2002). Context-dependent violations of rational choice in honeybees (Apis mellifera) and gray jays (Perisoreus canadensis). Behavioral Ecology and Sociobiology, 51, $180-187$.

Simonson, I. (1989). Choice based on reasons: The case of attraction and compromise effects. Journal of Consumer Research, 16, 158-174.

Smith, J. D., Coutinho, M. V. C., Church, B., \& Beran, M. J. (2013a). Executive-attentional uncertainty responses by rhesus monkeys 
(Macaca mulatta). Journal of Experimental Psychology: General, 142, 458-475. doi:10.1037/a0029601

Smith, J. D., Flemming, T. M., Boomer, J., Beran, M. J., \& Church, B. A. (2013b). Fading perceptual resemblance: A path for rhesus macaques (Macaca mulatta) to conceptual matching? Cognition, 129, 598-614.

Trueblood, J. S., Brown, S. D., Heathcote, A., \& Busemeyer, J. R. (2013). Not just for consumers: Context effects are fundamental to decision making. Psychological Science, 24, 901-908.
Tversky, A. (1969). Intransitivity of preferences. Psychological Review, 76, 31-48. doi:10.1037/h0026750

Tversky, A. (1972). Elimination by aspects: A theory of choice. Psychological Review, 79, 281-299. doi:10.1037/h0032955

Wedell, D. H. (1991). Distinguishing among models of contextually induced preference reversals. Journal of Experimental Psychology: Learning, Memory, and Cognition, 17, 767-778. doi:10.1037/ 0278-7393.17.4.767 